\title{
Association between Health Behaviors and a Family History of Cancer among Korean Women
}

\section{Youngsun Ham, $\mathrm{MScPH}^{1}$ \\ Hea Young $\mathrm{Oh}, \mathrm{PhD}^{2}$ \\ Sang-Soo Seo, MD, $\mathrm{PhD} \mathrm{D}^{3}$ \\ Mi Kyung Kim, PhD²}

${ }^{1}$ Berlin School of Public Health,

Charité - Universitätsmedizin Berlin,

Berlin, Germany,

${ }^{2}$ Division of Cancer Epidemiology and

Prevention, National Cancer Center, Goyang,

${ }^{3}$ Center for Uterine Cancer,

National Cancer Center, Goyang, Korea

\section{Purpose}

The aim of this study was to examine the health-related behaviors related to a family history of cancer (FHCA) among Korean women underwent cancer screening.

\section{Materials and Methods}

A total of 8,956 women who underwent cancer screenings during 2001-2011 at the National Cancer Center, Korea, were analyzed. The association between health-related behaviors and a FHCA were assessed using multivariate logistic regression.

\section{Results}

Compared to women with no FHCA, women with FHCA were more likely to smoke (adjusted odds ratio [aOR], 1.32; 95\% confidence interval [Cl], 1.06 to 1.65), to be exposed to passive smoking (aOR, 1.21; 95\% Cl, 1.15 to 1.65), and less likely to engage in regular exercise (aOR, 1.20; $95 \% \mathrm{Cl}, 1.01$ to 1.41). Combined effects of selected health behaviors for FHCA were significant, although no statistically significant interactions were observed between selected health behaviors. Compared to women with no FHCA, women with FHCA were more likely to simultaneously smoke and be exposed to passive smoking (aOR, 1.65; 95\% Cl, 1.17 to 2.31 ) and to simultaneously smoke and be physically inactive (aOR, 1.62; $95 \% \mathrm{Cl}$, 1.00 to 2.64$)$.

\section{Conclusion}

The study found that women with a FHCA exhibited unhealthy behaviors compared to women without FHCA. Higher emphasis on lifestyle modifications using a new standardized tool is strongly recommended for those with a FHCA, as well as individuals who are at high risk, together with their family members.
Correspondence: Mi Kyung Kim, PhD Division of Cancer Epidemiology and Prevention, National Cancer Center, 323 Ilsan-ro, Ilsandong-gu, Goyang 10408, Korea Tel: 82-31-920-2202

Fax: 82-31-920-2006

E-mail: alrud@ncc.re.kr

Received February 27, 2015

Accepted September 5, 2015

Published Online September 22, 2015

\section{Key words}

Health behavior, Early detection of cancer, Prevention and control, Family history of cancer

\section{Introduction}

A number of studies have investigated the relationship between risk factors and the prevalence of common chronic diseases. According to those studies, development of most chronic diseases such as cardiovascular disease, diabetes type 2 , and stroke, occurs as a result of complicated interactions between genetic, environmental, and lifestyle factors [1]. Cancer is also a chronic disease, strongly associated with modifiable lifestyle factors, such as obesity, physical inactivity, smoking, heavy alcohol intake, and dietary habit $[2,3]$.

Family history of cancer (FHCA) is one of several crucial risk factors for cancers. For example, development of breast cancer is two times more likely for women who have first degree relatives with breast cancer [4], and the risk for development of gastric cancer is three-fold for individuals with a positive family history of gastric cancer [5]. In addition, $20 \%$ of patients diagnosed with colorectal cancer (CRC) had a family history of the disease, but only $5 \%$ of them were 
single-gene disorders [6]. Thus, because FHCA reflects heredity, shared familiar environment, and behaviors of patients, a FHCA has been considered a critical risk factor for development of cancer [7].

Because FHCA has been considered an important risk factor for cancer, cancer prevention programs for the general population have included information about a FHCA and its impact, and have provided education regarding healthy behaviors, including cancer screening, to the entire population. Several studies have compared the health-related behaviors of people with and without FHCA. Most studies have reported that people with a FHCA were more likely to undergo cancer screening as a primary prevention tool; however, those participants did not significantly engage in healthy behaviors such as quitting smoking, maintaining a normal body mass index (BMI), or performing regular physical exercise [8]. However, little is understood of its impacts on individuals' health behaviors, even when individuals are aware of preventive behaviors against cancer and their FHCA status.

Because a FHCA is a well-known risk factor for cancers, individuals with a FHCA may be more likely to engage in healthy behaviors such as avoiding cigarette smoking, maintaining a normal body weight, or performing regular physical exercise. This study examined health-related behaviors (cigarette smoking, passive smoking, alcohol drinking, maintaining a normal body weight, or performing regular physical exercise) of Korean women who reported having one or more family members with cancer, compared with health behaviors of individuals who report no FHCA among cancer screening examinees at the National Cancer Center (NCC) in Korea. This study investigated the hypothesis that women with a FHCA would exhibit healthier behaviors, compared to those without a FHCA.

\section{Materials and Methods}

\section{Study subjects}

The study population was recruited at the National Cancer Control Institute, NCC in Korea. Data were collected from women who underwent cancer screenings between June 2001 and April 2011. The study protocol was approved by the Institutional Review Boards of the NCC in Korea (NCCNCS-1110320). Written informed consent was obtained from each study participant. During cancer screenings, study participants were asked to fill in structured questionnaires composed of the following components; socio-demographic characteristics (e.g., age, education level, occupation, family income, marital status, and place of residence), health-related characteristics (e.g., smoking habits, passive smoking, alcohol intake, diet, exercise, participation in health check-ups, menopause status, birth control use, and breastfeeding), personal medical and disease history, FHCA, and undergoing cancer screenings.

Study subjects $(n=9,506)$ who marked the question regarding a FHCA were included in the study population. If individuals participated in cancer screening more than one time, first data among several examinations were included in this analysis. All degrees of family relationship (first-degree: parents, siblings, and children; second-degree: grandparents, aunts and uncles; and other degree: cousins, nephews, and the other biological relatives) and all types of cancer history were considered. To avoid bias, 550 women with a personal history of any cancer were excluded. In total, 8,956 women were selected for the analysis, and of these, 5,281 had a FHCA.

\section{Measures}

The following variables were examined age, monthly family income, education level, BMI, number of children, menopause status, smoking, passive smoking, alcohol intake, regular physical activity, family history of any cancer, and family histories of cancer types among women with a FHCA. Information on smoking habits, alcohol intake, and exposure to passive smoke was collected across three categories: current, former, and never. For this study, former and never users were reclassified as non-users. Only current users were considered as the categories of drinker, smoker, and passive smoker. The variable of passive smoking included both family and workplace exposure. BMI was calculated using patients' height and weight measured during cancer screening at the NCC. For logistic regression, BMI was categorized according to two levels: non-overweight (less than $23 \mathrm{~kg} / \mathrm{m}^{2}$, normal, and underweight) and overweight (more than $23 \mathrm{~kg} / \mathrm{m}^{2}$, overweight, and obesity). This standard followed the Asian population criteria (underweight, $<18.5 \mathrm{~kg} / \mathrm{m}^{2}$; normal range, $18.5-22.99 \mathrm{~kg} / \mathrm{m}^{2}$; overweight, $23-24.99 \mathrm{~kg} / \mathrm{m}^{2}$; and obesity, $\left.\geq 25 \mathrm{~kg} / \mathrm{m}^{2}\right)[9,10]$. Height and weight of participants were measured at the NCC to avoid bias due to selfreport. The question regarding regular physical activity asked whether or not the respondents were exercising regularly. This question was not asked from 2004 to 2007 due to revised versions of questionnaires. The continuous age variable was converted into categorical variables of five levels (below 30, 30-39, 40-49, 50-59, and 60 and higher). Monthly family income was initially collected as Korean currency, but it was recalculated to approximate US dollars for this study. We examined two variables of FHCA according to two categories: 1, family history of any cancer; 2, FHCA for each can- 
cer site (stomach, colorectal, liver, lung, breast, cervical, and other cancers). These seven categories of cancer type were recoded according to the criteria of the Korean Central Cancer Registry [11]. FHCA sites included all degrees of history.

\section{Statistical analysis}

Statistical significance was assessed by chi-square test with the following demographic variables: age (less than 30, 30-39, 40-49, 50-59, and 60 and higher), education level (less than high school, high school, and higher than high school), family monthly income (less than 1,000, 1,000-1,990, 2,0003,990, 4,000-6,990, and higher than 7,000 USD), BMI (underweight, $<18.5 \mathrm{~kg} / \mathrm{m}^{2}$; normal, $18.5-23 \mathrm{~kg} / \mathrm{m}^{2}$; overweight, $23-25 \mathrm{~kg} / \mathrm{m}^{2}$; and obesity, $\left.\geq 25 \mathrm{~kg} / \mathrm{m}^{2}\right)$, number of children $(0,1-2,3-4$, and 5-9), and menopausal status (no and yes).

To examine the association between health behaviors in relation to a FHCA, multiple logistic regression was performed with the following five health behavior variables: (1) smoking (current vs. non-smoking), (2) passive smoking (yes vs. no), (3) alcohol intake (current vs. non-drinking), (4) BMI (non-overweight $<23 \mathrm{~kg} / \mathrm{m}^{2}$ vs. overweight $\geq 23 \mathrm{~kg} / \mathrm{m}^{2}$ ), and (5) regular daily exercise (yes vs. no). Selected dependent variables consisted of two categories: (1) family history of any cancer (first-degree, second-degree, and other-degree), (2) six major cancers (stomach, colorectal, liver, lung, breast, and cervical), and other cancers. Odds ratios (ORs) and 95\% confidence intervals (CIs) of FHCA and six major cancers were estimated with no family history of any cancer as a reference category. All variables in this analysis were adjusted for age, family monthly income, education, smoking status, passive smoking status, alcohol drinking status, BMI, the number of children, job status (no and yes), and history of chronic diseases (no and yes), as categorical types. Cochran Mantel-Haenszel tests were also performed for homogeneity of the associations with selected health behaviors across cancer screening year by fitting a model using ordinal values. $\mathrm{p}$-values were all more than 0.05 , then the analyses were performed in combined dataset by year.

To assess the combined effect of smoking, passive smoking, alcohol intake, BMI $\geq 23 \mathrm{~kg} / \mathrm{m}^{2}$, and exercise, multivariate logistic regression was performed on the additive scaled model of the health behaviors: e.g., no smoking and no alcohol drinking (OR1), smoking and no alcohol drinking (OR2), no smoking and alcohol drinking (OR3), and smoking and alcohol drinking (OR4). ORs and 95\% CIs were estimated with the combination of no smoking and no alcohol drinking (OR1) as a reference category. All variables were adjusted for the previously indicated covariates. The $\mathrm{p}$ for interaction was determined by multivariate logistic regression analysis for a multiplicative term (ex., smoking $\times$ alcohol drinking). The $p$ for homogeneity was determined using the Mantel-Haenszel test for ORs of smoking or passive smoking when stratified according to the status of passive smoking, alcohol intake, $\mathrm{BMI} \geq 23 \mathrm{~kg} / \mathrm{m}^{2}$, and exercise. All analyses were performed using SPSS ver. 20.0 (IBM Co., Armonk, NY) and STATA ver. 12.0 (StataCorp., College Station, TX).

\section{Results}

\section{FHCA among cancer screening examinee}

Table 1 shows demographic variables between women with and without a FHCA. Of a total of 8,956 subjects in the study population, $59 \%$ had a family history of any cancer, $20 \%$ for stomach cancer $(n=1,822)$. Cervical cancer was the least prevalent type of cancer in the group of women with a FHCA $(6 \%, n=576)$. Regarding age, nearly $40 \%$ of women were 40 to 49 years old, and less than $1 \%$ were under 30 . Approximately $80 \%$ of women had at least completed high school. On average, less than $20 \%$ of women had not completed high school, whereas more than $40 \%$ of women had some university-level education. However, women with relatives with breast and colorectal cancers had a higher education level $(52 \%, \mathrm{p}<0.01)$ than other cancer sites, while only $38 \%$ of women with no FHCA had college/ university degrees. Approximately $84 \%$ of women had more than 2,000 US dollars of monthly family income. More than $20 \%$ of women in each cancer site were recorded as obese, i.e., $\geq 25$ $\mathrm{kg} / \mathrm{m}^{2}$. In particular, women with no FHCA showed higher obesity rates $(23 \%)$ than women with a family history of other cancer sites. Approximately $62 \%$ of participants had one or two children, and nearly $13 \%$ had no children. However, $24 \%$ of women with a family history of cervical cancer had no children. The ratio of women who had reached menopause was approximately $57 \%$ in women with no FHCA and 51\% in women with a FHCA $(p<0.01)$.

\section{Association between health behaviors and a FHCA}

Compared to women with no FHCA, women with FHCA were more likely to smoke (adjusted odds ratio [aOR], 1.32; 95\% CI, 1.06 to 1.65), to be exposed to passive smoking (aOR, $1.21 ; 95 \% \mathrm{CI}, 1.15$ to 1.65$)$, and less likely to engage in regular exercise (aOR, 1.20; 95\% CI, 1.01 to 1.41) (Table 2). Women with a family history of colorectal or cervical cancer were more likely to smoke (colorectal cancer: aOR, 1.90; 95\% CI, 1.33 to 2.72; cervical cancer: aOR, 1.66; $95 \%$ CI, 1.10 to 2.51; other cancers: aOR, 1.53; 95\% CI, 1.15 to 2.03), and those with a family history of stomach, liver or lung cancer were more likely to be exposed to passive smoking (stomach: aOR, 1.39; 


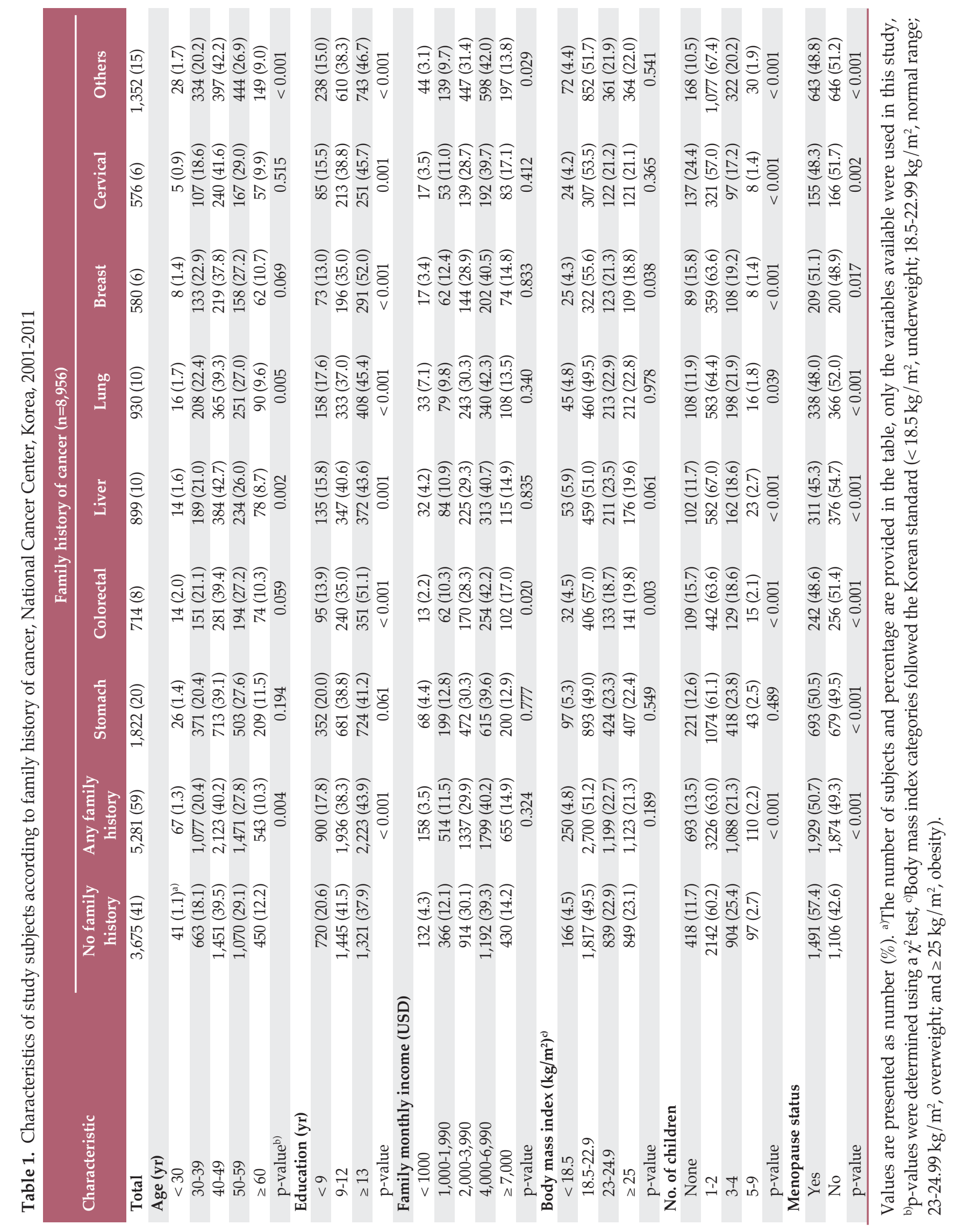




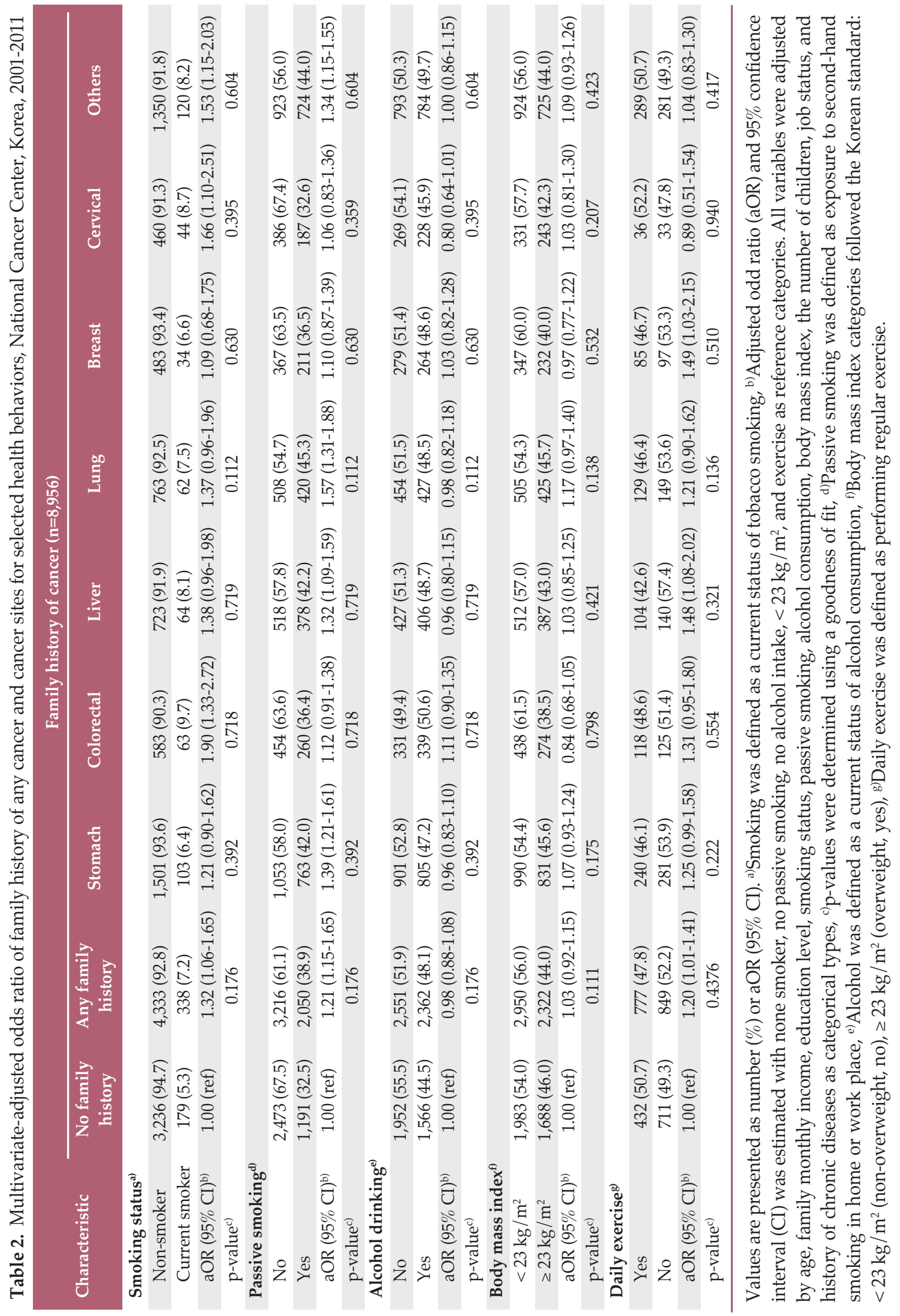


95\% CI, 1.21 to 1.61; liver: aOR, 1.32; 95\% CI, 1.09 to 1.59 ; lung: aOR, 1.57; 95\% CI, 1.31 to 1.88; other cancers: aOR, 1.34; 95\% CI, 1.15 to 1.55). Women with a family history of liver or breast cancer were physically inactive (liver: aOR, 1.48; 95\% CI, 1.08 to 2.02; breast: aOR, 1.49; 95\% CI, 1.03 to 2.15).

The combined associations of selected health behaviors with the FHCA are shown in Table 3. Overall, we found a combined effect of selected health behaviors for the FHCA, although there were no statistically significant interactions. Compared to women with no FHCA, women with a FHCA were more likely to simultaneously smoke and be exposed to passive smoking (aOR, 1.65; 95\% CI, 1.17 to 2.31 ) and to simultaneously smoke and be physically inactive (aOR, 1.62; $95 \% \mathrm{CI}, 1.00$ to 2.64). Although alcohol drinking and obesity, respectively, were not significantly associated with the FHCA, combined effect of smoking and passive smoking showed significant association.

\section{Discussion}

The aim of this study is to test the hypothesis that individuals at risk due to a FHCA are more health-conscious and may be more likely to engage in healthy behaviors such as avoiding cigarette smoking, maintaining a normal body weight or performing regular physical exercise. Contrary to our hypothesis, we found that women with any FHCA were more likely to smoke and to be exposed to passive smoking, and less likely to engage in regular exercise than those without a FHCA. In addition, women with a family history of liver or breast cancer were more physically inactive than those without. Women with a family history of colorectal or cervical cancer were more likely to smoke, and those with a family history of stomach, liver, or lung cancer were more likely to be exposed to passive smoking, compared to women with no FHCA. Previous studies found that study subjects were not less likely to maintain healthy behaviors, such as physical activity, dietary habits, smoking, and alcohol consumption $[8,12-14]$. In some of these studies, findings were drawn from women of higher socioeconomic status or a single institute [12], these findings may not be generalizable to the entire or other populations, including ours. Although our finding of health behavior (smoking, obesity, physical activity, and alcohol consumption) in relation to FHCA are generally similar to those from other recent studies [8,12-14], our finding on passive smoking appears to be novel. To the best of our knowledge, this finding is among of the first to be presented for individuals with an FHCA. In addition, this finding strengthens the evidence on all health behaviors and FHCA among women with cancer screening.
Our study population consisted of individuals who underwent cancer screening, meaning they would be more aware of cancer prevention and have more knowledge of prevention practices as compared to the general population. To verify this assumption, we examined the following features of our study population: family income level, level of education, and rate of FHCA. First, this population has a higher level of income than the general population [15]. According to two studies investigating the relationship between healthier behaviors and socio-demographic factors in the general population in Korea, higher-income men and women showed healthier behaviors, such as participation in cancer screening [16] and physical activity, compared to lower income populations $[16,17]$. Second, our study subjects had a higher education level than the general population (Table 1) [17]. According to a Korean population study, individuals younger than 49 years with a post-high school education level were more likely to seek cancer information [18]. Consequently, one can assume that awareness of healthy lifestyle behaviors is more prevalent in this study population than in the general population. Therefore, conduct of the study with this special population would be worthwhile for assessment of our current cancer prevention programs in Korea.

Taken together, our findings do not support our hypothesis that individuals with a FHCA may show more protective behaviors as compared to those without, although they were aware of a FHCA. Some studies have found that only $5 \%$ of CRC cases among people with a FHCA were associated with a single-gene mutation [6], indicating that most cancer cases develop by multifactorial interaction, such as smoking, heavy alcohol drinking, physical inactivity, larger waist circumference, obesity, and genetic factors. As study revealed, our population with a FHCA may also be more likely to be exposed to a higher risk environment, which leads to obtaining cancer in their families. This assumption could be well explained by passive smoking [19], because exposure to secondary smoking in families in Korea is strongly related to familiar environment, particularly for housewives due to traditional cultural background, such as patriarchal Confucianism.

A misconception about family history is also a possible reason that people do not change their risky behaviors to healthier ones. A study conducted in the United States assessing individuals' perceptions regarding the cause of cancer found that a majority of women considered heredity the most important factor in development of breast and CRC $(84.4 \%$, $78.5 \%)$, followed by lifestyle behaviors such as unhealthy dietary habits $(46.4 \%, 69.7 \%)$, smoking $(58.3 \%, 50.8 \%)$, and physical inactivity $(35.7 \%, 44.3 \%)$ [20]. However, for example, only $20 \%$ of CRC cases are actually related to a FHCA [6], that is, all other cases are associated with environmental or lifestyle risk factors that are amenable to intervention [21]. 


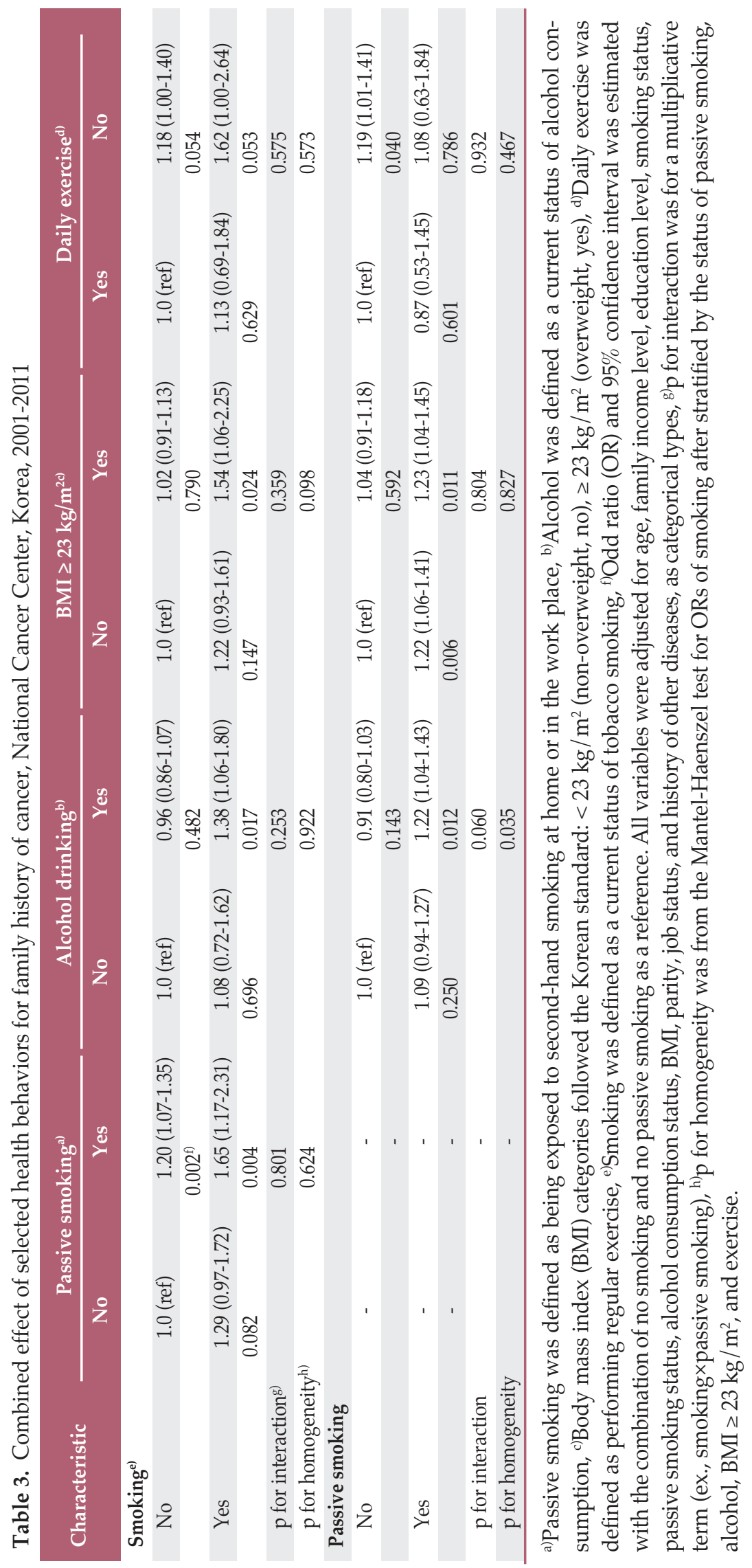


Regarding that misconception, the reason people strongly believe that heredity of cancer rather than lifestyle factors are causes of development of cancer has not yet been elucidated. One assumption is that people may tend to think that "there is nothing you can do about it" because they look upon diseases caused by genes as uncontrollable [22]. According to a recent study, women are likely to consider personal behavior or lifestyle as largely irrelevant to the development of cancer (OR, 1.24; 95\% CI, 1.01 to 1.52) [23]. This common perception might also explain why the participants in our study did not engage in lifestyle modification despite their FHCA state.

There are other possible explanations as to why people who are aware that they are at higher risk of developing cancer, may not engage in lifestyle modification. One explanation for this lifestyle rigidity derives from the psychological hypothesis of "choice overload," a theory which states that too much choice may lead to indecisiveness [24]. The causes of any cancer are often multifactorial, and the general population has been educated on all possible causes and how to avoid cancer. For example, development of CRC may be associated with a number of interacting genetic and lifestyle factors such as over-consumption of red and processed meat, overweight, or smoking [21]. Therefore, the choices of healthy lifestyle modifications might be overwhelming. One must maintain a normal range of weight, healthy regulation of red meat and alcoholic intake. In addition, cancer can be cured, if it is detected at an early stage, so that populations at high risk of cancer may consider cancer screenings as their best preventive option in terms of cost-effectiveness.

Another potential explanation for the low level of adherence to healthy lifestyle options among women with a FHCA in our study population was restricted focus of the National Cancer Control Program (NCCP) on cancer-screening programs with lower priority given to education campaigns. For example, when patients with a FHCA see doctors, clinicians advocated more regular cancer screening rather than encouraging patients to change lifestyles, and cancer screening is common for the general population in Korea. This is because it is an easier way to persuade patients to undergo cancer screenings within a short time than to encourage patients to change their lifestyle. Cancer screening is the most effective method for detection of cancer; however, this might not be a tool for primary prevention of cancer. Therefore, lifestyle modifications should be the first consideration for the public. Thus, the following is suggested: clinicians and patients with a FHCA should perceive that a FHCA is not just a cause of developing cancers, but it is a warning sign to those with fixed unhealthy behaviors, which might be changed. In addition, there is a need for development of standardized tools / guidelines, which can be useful in collection of information on FHCA from the patients and in providing them with accurate information as a primary prevention setting
[25].

The results of this study had limitations. Subjects included in this study were limited only to a single institute and to women, therefore findings from this study may not be generalized to the entire Korean population. Nevertheless, this study comprised relatively larger samples, and potential effects of confounding variables including age, family income, education, occupation, and a personal history of other chronic diseases were examined. FHCA was not verified against medical records or cancer registry data, thus under- or over-reporting remained in this analysis.

\section{Conclusion}

In conclusion, this study found that women with a FHCA did not show healthier behaviors compared to women without a FHCA, although a FHCA is among the well-known risk factors of cancers. FHCA indicates a large amount of information in terms of genetic, environmental, and behavioral factors that can cause cancer. Hence, our findings suggest that providing accurate knowledge regarding the implications of a FHCA by health professionals and the media should be provided to the general population at the primary intervention level. Therefore, it is necessary to build a new standardized tool for individuals with a FHCA to provide accurate information and to be able to engage in healthier behaviors for prevention of cancers. This provides a strong rationale for emphasizing lifestyle modifications which can be followed by individuals at high risk of developing cancer, and by population level.

\section{Conflicts of Interest}

Conflict of interest relevant to this article was not reported.

\section{Acknowledgments}

This research was in part supported by a National Cancer Center Grant (NCC1110320, NCC1310361, NCC-0910220) by the National Cancer Center, Republic of Korea. 


\section{References}

1. Strong K, Mathers C, Epping-Jordan J, Beaglehole R. Preventing chronic disease: a priority for global health. Int J Epidemiol. 2006;35:492-4.

2. Giovannucci E, Ascherio A, Rimm EB, Colditz GA, Stampfer MJ, Willett WC. Physical activity, obesity, and risk for colon cancer and adenoma in men. Ann Intern Med. 1995;122: 327-34.

3. Friedenreich CM. Physical activity and cancer prevention: from observational to intervention research. Cancer Epidemiol Biomarkers Prev. 2001;10:287-301.

4. Pharoah PD, Day NE, Duffy S, Easton DF, Ponder BA. Family history and the risk of breast cancer: a systematic review and meta-analysis. Int J Cancer. 1997;71:800-9.

5. Fock KM, Talley N, Moayyedi P, Hunt R, Azuma T, Sugano $\mathrm{K}$, et al. Asia-Pacific consensus guidelines on gastric cancer prevention. J Gastroenterol Hepatol. 2008;23:351-65.

6. Ahsan H, Neugut AI, Garbowski GC, Jacobson JS, Forde KA, Treat MR, et al. Family history of colorectal adenomatous polyps and increased risk for colorectal cancer. Ann Intern Med. 1998;128:900-5.

7. Gail MH, Brinton LA, Byar DP, Corle DK, Green SB, Schairer $\mathrm{C}$, et al. Projecting individualized probabilities of developing breast cancer for white females who are being examined annually. J Natl Cancer Inst. 1989;81:1879-86.

8. Huang XE, Hirose K, Wakai K, Matsuo K, Ito H, Xiang J, et al. Comparison of lifestyle risk factors by family history for gastric, breast, lung and colorectal cancer. Asian Pac J Cancer Prev. 2004;5:419-27.

9. Townsend JS, Steele CB, Richardson LC, Stewart SL. Health behaviors and cancer screening among Californians with a family history of cancer. Genet Med. 2013;15:212-21.

10. World Health Organization Western Pacific Region. The AsiaPacific perspective: redefining obesity and its treatment. Balmain, NSW: Health Communications Australia Pty Limited; 2000.

11. Ministry of Health and Welfare. Annual report of cancer statistics in Korea in 2010. Seoul: Ministry of Health and Welfare; 2012.

12. Kang JM, Shin DW, Kwon YM, Park SM, Park MS, Park JH, et al. Stomach cancer screening and preventive behaviors in relatives of gastric cancer patients. World J Gastroenterol. 2011; 17:3518-25.

13. Matsubara H, Hayashi K, Sobue T, Mizunuma H, Suzuki S. Association between cancer screening behavior and family history among Japanese women. Prev Med. 2013;56:293-8.
14. Kaphingst KA, Lachance CR, Condit CM. Beliefs about heritability of cancer and health information seeking and preventive behaviors. J Cancer Educ. 2009;24:351-6.

15. Ministry of Health and Welfare. Korea Health Statistics 2011; Korea National Heath and Nutrition Exymination Survey (KNHANES V-2). Seoul: Ministry of Health and Welfare; 2011.

16. Park MJ, Park EC, Choi KS, Jun JK, Lee HY. Sociodemographic gradients in breast and cervical cancer screening in Korea: the Korean National Cancer Screening Survey (KNCSS) 20052009. BMC Cancer. 2011;11:257.

17. Lee K, Lim HT, Hwang SS, Chae DW, Park SM. Socio-economic disparities in behavioural risk factors for cancer and use of cancer screening services in Korean adults aged 30 years and older: the Third Korean National Health and Nutrition Examination Survey, 2005 (KNHANES III). Public Health. 2010;124:698-704.

18. Kye SY, Cho J, Noh HI, Kwak MS, Chang YJ. What types of cancer screening information are needed? Asian Pac J Cancer Prev. 2010;11:607-13.

19. Lee BE, Ha EH. Exposure to environmental tobacco smoke among South Korean adults: a cross-sectional study of the 2005 Korea National Health and Nutrition Examination Survey. Environ Health. 2011;10:29.

20. Wang C, Miller SM, Egleston BL, Hay JL, Weinberg DS. Beliefs about the causes of breast and colorectal cancer among women in the general population. Cancer Causes Control. 2010;21: 99-107.

21. World Cancer Research Fund; American Institute for Cancer Research. Food, nutrition, physical activity, and the prevention of cancer: a global perspective. Washington, DC: American Institute for Cancer Research; 2007.

22. Marteau TM, Weinman J. Self-regulation and the behavioural response to DNA risk information: a theoretical analysis and framework for future research. Soc Sci Med. 2006;62:1360-8.

23. Kowalkowski MA, Hart SL, Du XL, Baraniuk S, Latini DM. Cancer perceptions: implications from the 2007 Health Information National Trends Survey. J Cancer Surviv. 2012;6: 287-95.

24. Scheibehenne B, Greifeneder R, Todd PM. Can there ever be too many options? A meta-analytic review of choice overload. J Consum Res. 2010;37:409-25.

25. Fiederling J, Ulrich CM, Hemminki K, Haug U. Consideration of family history of cancer in medical routine: a survey in the primary care setting in Germany. Eur J Cancer Prev. 2014; 23:199-205. 